\title{
A case study of mesospheric gravity wave momentum flux and dynamical instability using the Arecibo dual beam incoherent scatter
} radar

\author{
Qihou Zhou ${ }^{1}$ and Y. T. Morton ${ }^{1}$
}

Received 23 December 2005; revised 7 March 2006; accepted 5 April 2006; published 18 May 2006.

[1] We report the first observation of gravity wave momentum flux in the mesosphere using the dual-beam Arecibo incoherent scatter radar (ISR). Quasimonochromatic waves were observed throughout the daytime of July 28,2001 in the altitude range of $65-85 \mathrm{~km}$. The largest wave speed was about $100 \mathrm{~m} / \mathrm{s}$ and the dominant period was about $15 \mathrm{~min}$. Instability, as indicated by the Richardson number, occurred at several altitudes, most dramatically at 77 and 81 kilometers. The zonal momentum flux is found to change sign at altitudes where dynamical instability occurs. No significant gravity waves were observed in the region having an eastward background wind, while they were ubiquitous when the background wind was westward. Citation: Zhou, Q., and Y. T. Morton (2006), A case study of mesospheric gravity wave momentum flux and dynamical instability using the Arecibo dual beam incoherent scatter radar, Geophys. Res. Lett., 33, L10802, doi:10.1029/ 2005GL025608.

\section{Introduction}

[2] Gravity waves are an important contributor to the energetics and dynamics of the mesosphere. An extensive review on the theoretical and observational aspects of middle atmosphere gravity wave studies is given by Fritts and Alexander [2003] and Hecht [2004]. In this paper, we are primarily concerned with the measurement of gravity wave momentum flux and its relationship to the occurrence of instabilities in the mesosphere. Most recent work in this regard include those of Collins and Smith [2004], Fritts et al. [2004], Espy et al. [2004], Tang et al. [2005], and Li et al. [2005]. These studies have used lidars and all sky imagers to study the connections between the damping/ breaking of gravity waves and atmospheric instabilities at high altitudes [Collins and Smith, 2004; Fritts et al., 2004] and middle latitudes [Li et al., 2005]. Espy et al. [2004]and Tang et al. [2005] combined radar wind and airglow imager measurements to study the seasonal variations of vertical fluxes of horizontal momentum. Reid [2004] used an MF radar to obtain the density normalized upward flux of zonal momentum for scales smaller than the radar pulse volume.

[3] Despite their versatility in ionospheric observations, incoherent scatter radars (ISR) have been regarded to be unsuitable for gravity wave studies because of the long integration time needed in the mesosphere. Using a new

\footnotetext{
${ }^{1}$ Electrical and Computer Engineering Department, Miami University, Oxford, Ohio, USA.
}

Copyright 2006 by the American Geophysical Union. 0094-8276/06/2005GL025608\$05.00 radar pulsing scheme, Zhou [2000] demonstrated that the Arecibo ISR could achieve a time-resolution suitable to study gravity waves at practically all frequencies. In this study, we present the first dual beam incoherent scatter radar observation of the D-region winds. The coplanar dual beams make it possible to measure gravity wave momentum flux for intensive gravity wave events. In the following section, we present the observational results, including the wind velocities, Richardson number, and momentum fluxes. In Section 3, we discuss momentum fluxes at different time scales, and how they are related to the background wind and the occurrence of instability.

\section{Observational Results}

[4] The observation was carried out between 9:30 and 17:45 LT on July 28, 2001 at the Arecibo Observatory $\left(18^{\circ} \mathrm{N}, 67^{\circ} \mathrm{W}\right)$. The D-region data acquisition and processing for the Arecibo line-feed system is described by Zhou [2000]. In the dual beam configuration, a total of $1.5 \mathrm{MW}$ of transmitting power was split between the line-feed and the Gregorian feed. For the present work, we transmitted an 88 $\mu$ s baud code with a $2 \mu$ s baud length to obtain a height resolution of $300 \mathrm{~m}$. Both feeds were parked at 5 degrees off zenith with the Gregorian pointing due east and line-feed due west in the geographical coordinate. Figure 1 shows the line-of-sight velocity for the two feeds with the upward as the positive direction. The line of sight velocity error is slightly below $1 \mathrm{~m} / \mathrm{s}$ at $75 \mathrm{~km}$ and its variation as a function of height is given by Zhou [2000]. The region of valid measurements is largely confined to the altitude range of 65 to $85 \mathrm{~km}$. Below $65 \mathrm{~km}$, the ISR cannot make valid measurements because of lack of ionization. Above $85 \mathrm{~km}$, the incoherent scatter spectra become too wide to distinguish any spectral features.

[5] Assuming that the horizontal wavelength of the gravity waves is much larger than the distance between the dual beams, the horizontal and vertical velocity of a gravity wave can be calculated from the following equations:

$$
\begin{gathered}
V_{H}=\left.\frac{v_{1} \cos \alpha_{2}-v_{2} \cos \alpha_{1}}{\cos \alpha_{1} \sin \alpha_{2}+\sin \alpha_{1} \cos \alpha_{2}}\right|_{\alpha_{1}=\alpha_{2}=\alpha}=\frac{v_{1}-v_{2}}{2 \sin \alpha} \\
V_{z}=\left.\frac{v_{1} \sin \alpha_{2}+v_{2} \sin \alpha_{1}}{\cos \alpha_{1} \sin \alpha_{2}+\sin \alpha_{1} \cos \alpha_{2}}\right|_{\alpha_{1}=\alpha_{2}=\alpha}=\frac{v_{1}+v_{2}}{2 \cos \alpha}
\end{gathered}
$$

where $v_{1}$ and $v_{2}$ are the line of sight velocities at zenith angle $\alpha_{1}$ and $\alpha_{2}$, respectively. Line of sight velocity is positive if it is away from the radar and $\mathrm{v}_{\mathrm{z}}$ is positive upward. The positive direction of $\mathrm{V}_{\mathrm{H}}$ is the same as the 

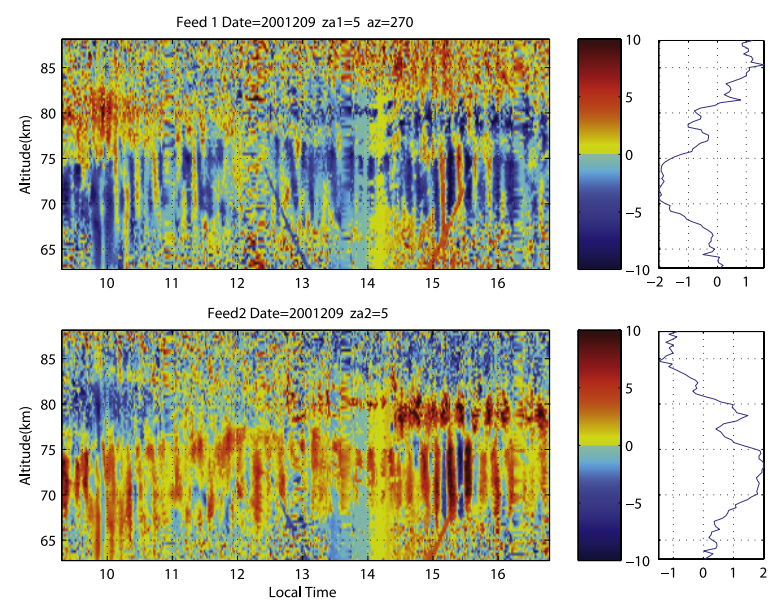

Figure 1. Line of sight velocities measured from the (top)line-feed and the (bottom) Gregorian feed of the Arecibo incoherent scatter radar on July 28, 2001. The linefeed and the Gregorian feed were pointed in the westward and eastward direction respectively. The zenith angle of both feeds was set at 5 degrees. Positive direction is away from the radar. The line-plot on the right is the mean velocity profile. The height and temporal resolution are $300 \mathrm{~m}$ and 66 seconds respectively.

projection of $\mathrm{v}_{1}$ on the horizontal plane. The simplified equations apply when the two feeds have the same zenith angle $\alpha$ but opposite azimuth angles.

[6] Figure 2 shows the eastward and upward velocity derived from the line-of-sight velocity using equations (1) and (2). One needs to bear in mind that the validity of the horizontal and vertical winds depends on the assumption that the horizontal wavelength is much larger than the horizontal beam separation distance, which was $14 \mathrm{~km}$ at $80 \mathrm{~km}$ altitude. One way to test this assumption is to examine the cross-correlation coefficient of the two lineof-sight velocities. Because the radar pointing is nearly vertical, the occurrence of the maximum negative correlation near the zero lag implies that the horizontal wavelength is much larger than the beam separation distance. Cross-

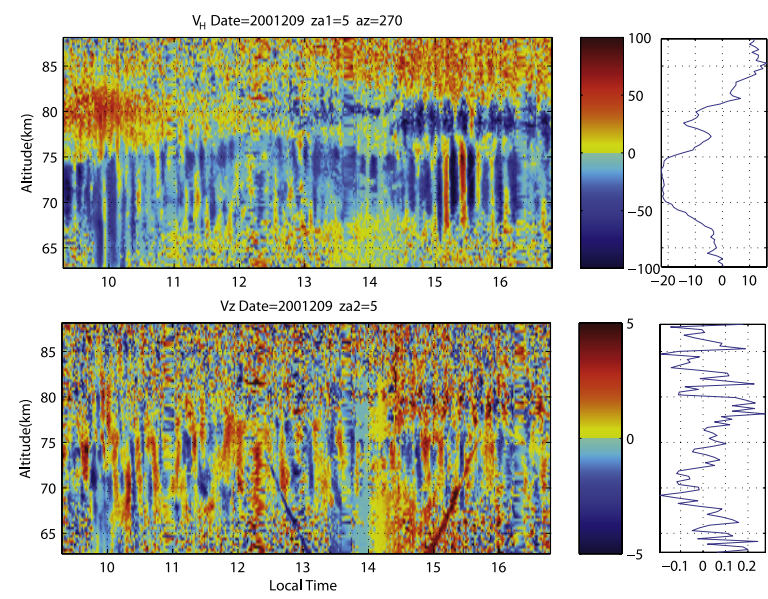

Figure 2. Eastward and upward wind velocities derived from the line-of-sight velocities shown in Figure 1. The line-plot on the right is the mean velocity profile. correlation analysis shows that the large horizontal wavelength assumption is largely valid. When the horizontal wavelength is not much larger than the beam separation, the derived velocity amplitude tends to be smaller than the true amplitude. We also note that the small beam illumination area, $300 \mathrm{~m}$ in diameter in the mesosphere, makes measurements of small gravity waves amplitudes more accurate than medium frequency (MF) and typical mesosphere-stratosphere-troposhere (MST) radars.

[7] The most striking feature in the velocities is the dominance of gravity waves having a period of about $15 \mathrm{~min}$ in the altitude range from about $65 \mathrm{~km}$ to $80 \mathrm{~km}$ all the time. In this $15 \mathrm{~km}$ altitude range, there is essentially no phase progression, indicating a very large vertical wavelength. The most intensive gravity wave occurred between 15:00 to 15:40 when its amplitude was as high as $100 \mathrm{~m} / \mathrm{s}$. From 14:20 to 16:45, there are two regions of strong gravity wave activities, one above and another below about $78 \mathrm{~km}$. The wave frequencies in these two regions are essentially the same but a phase reversal is clearly seen at around $78 \mathrm{~km}$. The phase reversal implies a very large wind shear, which may drive the atmosphere to be unstable. In the remainder of this study, we will focus on the period between 14:20 to 16:45 to examine the momentum flux and its relation with instability.

[8] A common criterion to gauge the stability of the atmosphere is the Richardson number $\left(\mathrm{R}_{\mathrm{i}}\right)$, which is defined as $\omega_{b}^{2} /(d U / d z)^{2}$, where $d U / d z$ is vertical gradient of horizontal velocity and $\omega_{\mathrm{b}}$ is the Brunt-Vaisala frequency. Figure 3 shows the calculated Richardson number using the measured zonal wind shown in Figure 2. In calculating $\omega_{\mathrm{b}}$, we have used the MSIS (Mass Spectrometer and Incoherent Scatter) atmospheric temperature profile. A region having a $R_{i}$ value smaller than 0.25 is generally regarded to be dynamically unstable [e.g., Hecht, 2004]. Figure 3 shows that pockets of unstable regions are centered at the altitudes of 77 and $81 \mathrm{~km}$, respectively. From Figures 1 and 2, it is seen that the wave event reverses its phase from $77 \mathrm{~km}$ to 81 $\mathrm{km}$. Since it takes several kilometers to complete the phase reversal, multiple layers of large shear during the phase transition can exist. Figure 3 indicates that the largest shears occur at the start and the end height of the phase transition,

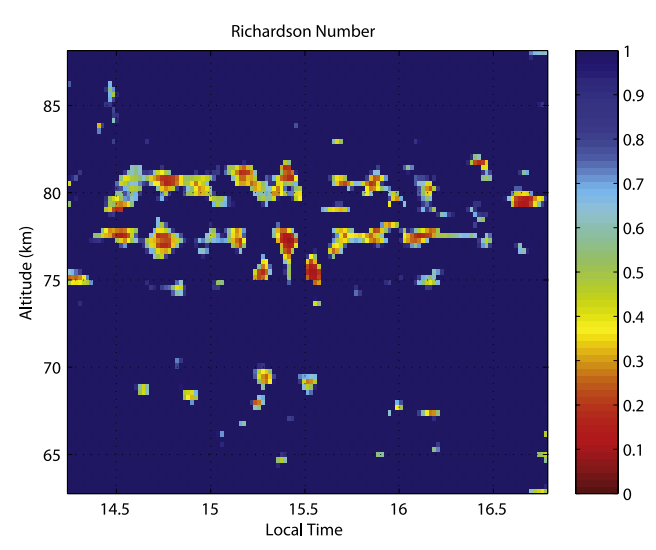

Figure 3. Richardson number calculated from the horizontal velocity shown in Figure 2. MSIS model temperature profile was used to compute the Brunt-Vaisala frequency. 
instead of in the middle of it. This result highlights the importance of fine height resolution in studying turbulent layers. We also note the unstable regions shown in Figure 3 are likely an underestimate of the true unstable regions because of the omission of meridional wind and the effect of temperature change.

[9] The dual beam capability at Arecibo makes it possible to measure the momentum flux per unit mass, or the covariance of horizontal and vertical winds. Despite the fact that long observation time is required for climatology studies [Kudeki and Franke, 1998], we can measure the momentum flux associated with individual intense gravity waves during a relative short period. As pointed out by Vicent and Reid [1983], the covariance of two symmetrical coplanar beams can be measured conveniently through the following equation:

$$
\langle u w\rangle=\frac{\left\langle v^{2}(\theta)\right\rangle-\left\langle v^{2}(-\theta)\right\rangle}{2 \sin (2 \theta)}
$$

where $\mathrm{v}(\theta)$ is the velocity at zenith angle $\theta$, and $\mathrm{u}$ and $\mathrm{w}$ are the horizontal and vertical wind, respectively. Angle bracket \langle\rangle indicates time average. Of great interest is the covariance of velocity perturbations, which can be expressed as:

$$
\left\langle u^{\prime} w^{\prime}\right\rangle=\frac{\left\langle[v(\theta)-\langle v(\theta)\rangle]^{2}\right\rangle-\left\langle[v(-\theta)-\langle v(-\theta)\rangle]^{2}\right\rangle}{2 \sin (2 \theta)}
$$

In the above expression, $\mathrm{u}^{\prime}$ and $\mathrm{w}^{\prime}$ represent horizontal and vertical velocity perturbations, respectively. In a physical sense, $\left\langle u^{\prime} w^{\prime}\right\rangle$ is a measure of the momentum flux associated with high frequency gravity waves, with the cut-off frequency dictated by the window length used to calculate the mean velocities.

[10] Figure 4 shows the two momentum fluxes, as expressed by equations (3) and (4), calculated for the period from 14:30 to 16:50. Included also is the mean horizontal and vertical wind for this period. In calculating the momentum fluxes, no smoothing was performed. Because of the height independence of the measured momentum fluxes, their altitude variation in a large degree reflects the precision of the measurements. The "error" bars for $\left\langle u^{\prime} w^{\prime}\right\rangle$, as shown in Figure 4, are the mean absolute differences between two adjacent heights. They are likely an overestimation of the true error because of the height variation of $\left\langle u^{\prime} w^{\prime}\right\rangle$. Even with the overestimation of the uncertainty, the trend of $\left\langle u^{\prime} w^{\prime}\right\rangle$ is clearly significant. It should be noted that one can also obtain the momentum flux uncertainty from the errors of the line-of-sight velocities, similar to what Liu and Gardner [2005] did for the heat-flux transfer. Such an estimate requires a good measurement of the velocity error itself and is not attempted here.

\section{Discussions}

[11] Since $\mathrm{w}=\langle\mathrm{w}\rangle+\mathrm{w}^{\prime}, \mathrm{u}=\langle\mathrm{u}\rangle+\mathrm{u}^{\prime}$, and with the assumptions that $\left\langle\mathrm{u}^{\prime}\right\rangle=\left\langle\mathrm{w}^{\prime}\right\rangle=0$, it follows that

$$
\langle u w\rangle=\langle u\rangle\langle w\rangle+\left\langle u^{\prime} w^{\prime}\right\rangle
$$

That is, the total momentum flux is the sum of the momentum flux of a low frequency component (back-

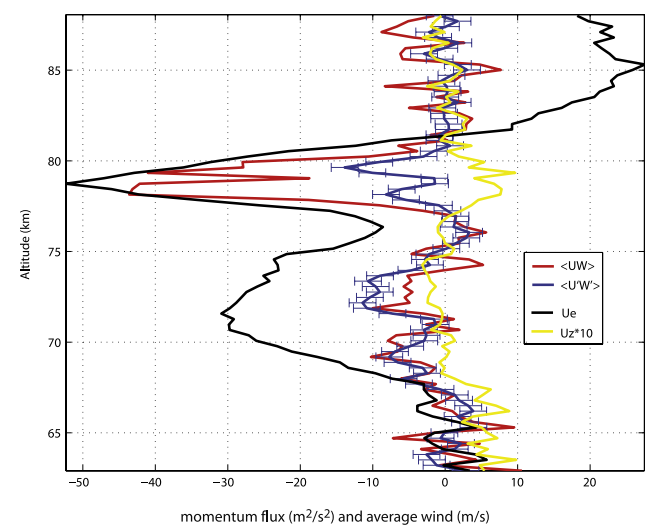

Figure 4. The red curve is the covariance of vertical and eastward wind without subtracting the averages. The blue curve is the covariance with the averages subtracted. The black curve is the average eastward wind and the yellow curve is 10 times of the vertical wind. All quantities were calculated for the period of 14:20 to 16:45, LT.

ground wind) and that of a high frequency component (gravity waves). Since the background wind in our case is averaged over a two hour period in our computation, it includes contributions from the mean wind, planetary waves, tides as well as long period gravity waves. Figure 4 shows that $\langle u w\rangle$ and $\left\langle u^{\prime} w^{\prime}\right\rangle$ largely overlap below $75 \mathrm{~km}$ where the mean vertical wind is very small. Around $78 \mathrm{~km}$, the magnitude of $\langle u w\rangle$ becomes as large as $40 \mathrm{~m}^{2} / \mathrm{s}^{2}$ while $\left\langle u^{\prime} w^{\prime}\right\rangle$, the momentum flux associated with high frequency gravity waves, remains about the same as that below $75 \mathrm{~km}$. The large total momentum flux is due to a strong mean upward and westward wind. Since $\langle u w\rangle$ from 9:30 to 12:00 is less than $5 \mathrm{~m}^{2} / \mathrm{s}^{2}$ around $78 \mathrm{~km}$, this indicates that the total momentum flux associated with the "background wind" during 14:30-16:50 is largely due to gravity waves having a period comparable to the window length of 2 hours.

[12] The mean zonal wind, as shown in Figure 4, is largely correlated with the momentum flux. The positive correlation between momentum flux and mean zonal wind was also reported by Riggin et al. [1997] using data taken by the Jicamarca radar in January 1993. However, the data taken at Jicamarca in Aug. 1994 reported by Fritts et al. [1997] showed a predominantly anti-correlation between zonal wind and momentum flux. According to Fritts et al., anti-correlation is generally expected until the remaining gravity waves in the spectrum encounter an opposite wind shear. Since the gravity waves in our observations are largely quasi-monochromatic, they are well filtered when reaching the region of our observations. The positive correlation may thus indicate that the gravity waves have gone through an opposite wind-shear at lower altitudes.

[13] Gravity wave theory suggests that the horizontal wave vector reverses direction when a gravity wave passes through a critical level at which the phase speed equals to the background wind speed [Nappo, 2002]. Since the direction of the momentum flux is related to the wave vector direction, reversing the horizontal wave vector (but not the vertical wave vector) direction would cause the reverse of the sign of the momentum flux. Sign change in momentum flux thus indicates a gravity wave going through 
a critical level. Figure 4 shows that gravity wave momentum flux changes sign or becomes zero at altitudes 67,75 , 77 , and $81 \mathrm{~km}$. All of these altitudes, especially the last two, are unstable, as seen from Figure 3. This is consistent with the gravity wave theory that the atmosphere typically becomes turbulent at critical levels. We further note that the Richardson number calculated for the entire observation from 9:30 to 16:45 indicates occurrence of dynamical instabilities at the altitudes where background wind changes from westward to eastward. This altitude moved up from about $74 \mathrm{~km}$ at 09:30 to $79 \mathrm{~km}$ at 14:00, consistent with previous observations of a turbulent layer at this altitude range [Zhou, 2000].

[14] Gravity wave filtering by the background wind is evident throughout the day. Figure 2 shows that gravity waves having a period of about 15 minutes are abundant in the region when the background wind is westward. Wherever the background wind was eastward, those short period gravity waves ceased to exist. We must caution, however, that this correlation does not necessarily mean that the filtering effect is by the zonal wind alone. Since it is unlikely that the gravity wave vectors are exactly within the zonal plane, filtering by the mean wind depends on the meridional wind as well. It is one of our main goals to obtain both components in future observations at Arecibo.

\section{Conclusion}

[15] We have presented the results from the first Arecibo dual-beam incoherent scatter radar observation. The dual beam results allowed us to calculate the momentum flux profile for an intense gravity wave having a maximum speed of about $100 \mathrm{~m} / \mathrm{s}$. The largest momentum flux, most likely due to gravity waves having a period of about two hours, was $40 \mathrm{~m}^{2} / \mathrm{s}^{2}$, and the momentum flux associated with high frequency component, at a period of about 15 minutes, was about $10 \mathrm{~m}^{2} / \mathrm{s}^{2}$. Dynamic instability was observed to occur at the altitudes where momentum flux changed sign. The background wind plays a very important role in filtering the gravity waves into quasi monochromatic waves. Zonal gravity waves in the mesosphere above Arecibo are largely filtered out by an eastward background wind. Our observation also showed that instability throughout the day occurred in the region where the background wind changed from a westward to an eastward direction. The current study demonstrates the potential of the Arecibo ISR for D-region gravity wave studies. It is clear, however, that multi-day observations are needed in order to delineate the characteristics of mesosphere gravity waves and their interactions with tides, planetary waves and the mean flows.

[16] The capability of the Arecibo ISR in characterizing gravity waves is currently being expanded in several areas. The daytime lidar being developed will allow direct measurements of temperature profile. Because of the comprehensive measurement capabilities at ionosphere heights, gravity wave penetration can be studied from the mesosphere to thermosphere, as demonstrated by Djuth et al. [2004]. These capabilities enable the Arecibo facility to be used for extensive gravity wave studies in the coming years.

[17] Acknowledgments. The Arecibo Observatory is the major facility of the National Astronomy and Ionosphere Center, which is operated by Cornell University under a cooperative agreement with the National Science Foundation. The work was supported by NSF grant 0337245 to Miami University.

\section{References}

Collins, R. L., and R. W. Smith (2004), Evidence of damping and overturning of gravity waves in the Arctic mesosphere: $\mathrm{Na}$ lidar and $\mathrm{OH}$ temperature observations, J. Atmos. Sol. Terr. Phys., 66, 867-879.

Djuth, F. T., M. P. Sulzer, S. A. Gonzalez, J. D. Mathews, J. H. Elder, and R. L. Walterscheid (2004), A continuum of gravity waves in the Arecibo thermosphere?, Geophys. Res. Lett., 31, L16801, doi:10.1029/ 2003GL019376.

Espy, P. J., G. O. L. Jones, G. R. Swenson, J. Tang, and M. J. Taylor (2004), Seasonal variations of the gravity wave momentum flux in the Antarctic mesosphere and lower thermosphere, J. Geophys. Res., 109, D23109, doi:10.1029/2003JD004446.

Fritts, D. C., and M. J. Alexander (2003), Gravity wave dynamics and effects in the middle atmosphere, Rev. Geophys., 41(1), 1003, doi:10.1029/2001RG000106.

Fritts, D. C., et al. (1997), Equatorial dynamics observed by rocket, radar, and satellite during the CADRE/MALTED campaign: 2. Mean and wave structures, coherence, and variability, J. Geophys. Res., 102, 26,19126,216.

Fritts, D. C., B. P. Williams, C. Y. She, J. D. Vance, M. Rapp, F.-J. Lübken, A. Müllemann, F. J. Schmidlin, and R. A. Goldberg (2004), Observations of extreme temperature and wind gradients near the summer mesopause during the MaCWAVE/MIDAS rocket campaign, Geophys. Res. Lett., 31, L24S06, doi:10.1029/2003GL019389.

Hecht, J. H. (2004), Instability layers and airglow imaging, Rev. Geophys., 42, RG1001, doi:10.1029/2003RG000131

Kudeki, E., and S. J. Franke (1998), Statistics of momentum flux estimation, J. Atmos. Sol. Terr. Phys., 60, 1549-1553.

Li, T., C. Y. She, B. P. Williams, T. Yuan, R. L. Collins, L. M. Kieffaber, and A. W. Peterson (2005), Concurrent $\mathrm{OH}$ imager and sodium temperature/ wind lidar observation of localized ripples over northern Colorado, J. Geophys. Res., 110, D13110, doi:10.1029/2004JD004885.

Liu, A. Z., and C. S. Gardner (2005), Vertical heat and constituent transport in the mesopause region by dissipating gravity waves at Maui, Hawail $\left(20.7^{\circ} \mathrm{N}\right)$, and Starfire Optical Range, New Mexico $\left(35^{\circ} \mathrm{N}\right), J$. Geophys. Res., 110, D09S13, doi:10.1029/2004JD004965.

Nappo, C. (2002), An Introduction to Atmospheric Gravity Waves, Elsevier, New York.

Reid, I. M. (2004), MF radar measurements of sub-scale mesospheric momentum flux, Geophys. Res. Lett., 31, L17103, doi:10.1029/ 2003GL019200.

Riggin, D. M., D. C. Fritts, C. D. Fawcett, E. Kudeki, and M. H. Hitchman (1997), Radar observations of gravity waves over Jicamarca, Peru during the CADRE campaign, J. Geophys. Res., 102, 26,263-26,281.

Tang, J., G. R. Swenson, A. Z. Liu, and F. Kamalabadi (2005), Observational investigations of gravity wave momentum flux with spectroscopic imaging, J. Geophys. Res., 110, D09S09, doi:10.1029/2004JD004778.

Vincent, R. A., and I. M. Reid (1983), HF doppler measurements of mesospheric gravity wave momentum fluxes, J. Atmos. Sci., 40, $1321-1333$

Zhou, Q. H. (2000), Incoherent scatter radar measurements of mesospheric vertical winds, Geophys. Res. Lett., 27, 1803-1806.

Y. T. Morton and Q. Zhou, Electrical and Computer Engineering Department, Miami University, Oxford, OH 45056, USA. (zhouq@muohio. edu) 